\title{
Posttraumatic therapeutic vaccination with modified myelin self-antigen prevents complete paralysis while avoiding autoimmune disease
}

\author{
Ehud Hauben, ${ }^{1}$ Eugenia Agranov, ${ }^{1}$ Amalia Gothilf, ${ }^{1}$ Uri Nevo, ${ }^{1}$ Avi Cohen, ${ }^{2}$ Igor Smirnov, ${ }^{2}$ \\ Lawrence Steinman, ${ }^{3}$ and Michal Schwartz ${ }^{1}$
}

${ }^{1}$ Department of Neurobiology, The Weizmann Institute of Science, Rehovot, Israel
2Proneuron Biotechnologies Ltd., Kiryat Weizmann, Ness Ziona, Israel
3Department of Neurology, Stanford University School of Medicine, Stanford, California, USA
Address correspondence to: Michal Schwartz, Department of Neurobiology, The Weizmann Institute of Science,
76100 Rehovot, Israel. Phone: 972-8-9342467; Fax: 972-8-9344131; E-mail: michal.schwartz@weizmann.ac.il.

Received for publication March 29, 2001, and accepted in revised form June 18, 2001.

\begin{abstract}
Spinal cord injury results in a massive loss of neurons, and thus of function. We recently reported that passive transfer of autoimmune $T$ cells directed against myelin-associated antigens provides acutely damaged spinal cords with effective neuroprotection. The therapeutic time window for the passive transfer of $T$ cells was found to be at least 1 week. Here we show that posttraumatic $T$ cell-based active vaccination is also neuroprotective. Immunization with myelin-associated antigens such as myelin basic protein (MBP) significantly promoted recovery after spinal cord contusion injury in the rat model. To reduce the risk of autoimmune disease while retaining the benefit of the immunization, we vaccinated the rats immediately after severe incomplete spinal cord injury with MBPderived altered peptide ligands. Immunization with these peptides resulted in significant protection from neuronal loss and thus in a reduced extent of paralysis, assessed by an open-field behavioral test. Retrograde labeling of the rubrospinal tracts and magnetic resonance imaging supported the behavioral results. Further optimization of nonpathogenic myelin-derived peptides can be expected to lead the way to the development of an effective therapeutic vaccination protocol as a strategy for the prevention of total paralysis after incomplete spinal cord injury.
\end{abstract}

J. Clin. Invest. 108:591-599 (2001). DOI:10.1172/JCI200112837.

\section{Introduction}

Propagation of damage is a common occurrence after any CNS insult. Consequently, the outcome of incomplete spinal cord injury (ISCI) is far more severe than might have been expected from the immediate effect of the insult. This is because the injury not only involves primary degeneration of the directly injured neurons, but also initiates a self-destructive process that leads to secondary degeneration of neighboring neurons that escaped the initial insult (1-3). A great deal of research has been devoted to limiting the extent of secondary degeneration and thereby improving functional recovery from partial CNS injury (4-14).

We have reported in the past few years that passive immunization with $\mathrm{T}$ cells directed to CNS-associated myelin antigens leads to reduction of secondary degeneration $(4,15)$. Given these results, we formulated the concept of protective autoimmunity (16).

Protective autoimmunity is a relatively new concept. It refers to a benign autoimmune response that contributes to the maintenance and protection of injured neurons and the promotion of recovery after traumatic injury to the CNS $(4,15-21)$. The pathological aspects of autoimmunity in the CNS, leading to various autoimmune disorders, are well-characterized. Understanding the mechanism of the benign autoim- mune response to injury-associated self-antigens, however, is still in its infancy $(22,23)$. This response may be viewed as reminiscent of the response evoked by pathogen attack, in which recruitment of the immune system is considered essential. Mechanical or biochemical insults to the CNS do not involve pathogens, and thus recruitment of the adaptive immune system has not been considered relevant, as there seems to be no obvious need to mount a defense. Our observations suggest that the role of protective immunity that develops under nonpathogenic conditions (such as those occurring in CNS trauma) is possibly to halt the progressive degeneration $(17,18)$. However, the ability of individuals or strains to spontaneously mount such a $\mathrm{T}$ cell-mediated protective immunity was found to be genetically controlled and was associated with the ability to resist development of an autoimmune disease, when challenged by encephalitogenic myelin antigens (17). Because the protective immunity was found to be amenable to boosting by passive transfer of $T$ cells, we explore here the possibility of translating this approach into an active therapeutic vaccination.

The therapeutic time window for the transfer of $\mathrm{T}$ cells after ISCI was found to be at least 1 week; passive transfer of T cells 1 week after ISCI was as effective as immediate injection of the T cells (15). A protocol based on 
transfer of $\mathrm{T}$ cells for therapeutic purposes requires the use of autologous cells and their ex vivo expansion. The therapeutic time window, however, may not allow withdrawal of blood, isolation and expansion of cells, and reintroduction to the same individual.

In the present study, we explored the possibility of translating our finding of $\mathrm{T}$ cell-mediated neuroprotection into an active therapeutic vaccination, taking advantage of the fact that the $\mathrm{T}$ cell response after immunization has a rapid onset (3-5 days).

In selecting a protocol for active immunization, the choice of a suitable self-antigen is complicated by the selected antigen's possible potential for autoimmune destruction. The identity of the endogenous antigen that evokes the physiological neuroprotective immune response after different insults is not yet fully characterized. The antigen selected to boost this response must induce a protective autoimmune response without causing an autoimmune disease. Nonencephalitogenic peptides derived from identified self-proteins seem to be promising candidates. However, owing to the diversity of the HLA in humans, a self-protein sequence that will be universally nonencephalitogenic is not likely to be found.

In the present study, we also address questions relating to the choice of safe antigens and of the protocol for immunization with them.

\section{Methods}

Animals. Inbred adult Lewis or Sprague-Dawley (SPD) rats (10-12 weeks old, 200-250 g) were supplied by the Animal Breeding Center of The Weizmann Institute of Science. The rats were matched for age and weight in each experiment and housed in a light- and temperature-controlled room.

Antigens. Myelin basic protein (MBP) was prepared from the spinal cords of guinea pigs (4) or purchased from Sigma Chemical Co. (St. Louis, Missouri, USA). Spinal cord homogenate was prepared from autologous rat spinal cords homogenized in PBS (vol/vol). Modified (nonencephalitogenic) MBP peptides were derived from an encephalitogenic peptide, amino acids 87-99 of MBP, by replacing the lysine residue 91 with glycine (G91, kindly donated by L. Steinman) or the proline residue 96 with alanine (96A, synthesized at the Weizmann Institute of Science, Rehovot, Israel). All peptides used in the study had a purity of greater than $95 \%$, as confirmed by reversephase HPLC. Antigens were emulsified in equal volumes of incomplete Freund's adjuvant (IFA; Difco Laboratories, Detroit, Michigan, USA), CFA supplemented with 5 $\mathrm{mg} / \mathrm{ml}$ Mycobacterium tuberculosis (Difco Laboratories) (an amount that leads to severe symptoms of experimental autoimmune encephalomyelitis [EAE] in uninjured rats), or CFA with a low bacterial supplement $(0.5 \mathrm{mg} / \mathrm{ml}$ Mycobacterium butyricum; Difco Laboratories).

Spinal cord contusion or transection. Rats were anesthetized by intraperitoneal injection of Rompun (xylazine, 10 $\mathrm{mg} / \mathrm{kg}$; Vitamed, Israel) and Vetalar (ketamine, $50 \mathrm{mg} / \mathrm{kg}$; Fort Dodge Laboratories, Fort Dodge, Iowa, USA), and their spinal cords were exposed by laminectomy at the level of T8. One hour after induction of anesthesia, a 10g rod was dropped onto the laminectomized cord from a height of $50 \mathrm{~mm}$, using the NYU impactor (New York University, New York, New York, USA), a device shown to inflict a well-calibrated contusive injury of the spinal cord $(5,6,15,24)$. The spinal cords of another group of rats were completely transected, as described previously (25).

Active immunization. Rats were immunized subcutaneously at the base of the tail, on a random basis, with MBP, spinal cord homogenate, or altered peptide ligands (APLs), or were injected with PBS, each emulsified in an equal volume of CFA containing $5 \mathrm{mg} / \mathrm{ml} M$. tuberculosis, CFA containing $0.5 \mathrm{mg} / \mathrm{ml}$ M. butyricum, or IFA. The immunization was performed within 1 hour after contusion or 7 days earlier. Control rats were sham-operated (laminectomized but not contused), immunized, and examined daily for symptoms of EAE, which were scored on a scale of 1 to 5 (26).

Animal care. In contused rats, bladder expression was assisted by massage at least twice a day (particularly during the first 48 hours after injury, when it was done three times a day), until the end of the second week, by which time automatic voidance had been recovered in SPD rats. Lewis rats required this treatment throughout the experiment. All rats were carefully monitored for evidence of urinary tract infection or any other sign of systemic disease. During the first week after contusion and in any case of hematuria after this period, they received a course of sulfamethoxazole $(400$ $\mathrm{mg} / \mathrm{ml})$ and trimethoprim $(8 \mathrm{mg} / \mathrm{ml}$ ) (Resprim; Teva Laboratories, Israel), administered orally with a tuberculin syringe ( $0.3 \mathrm{ml}$ of solution per day). Daily inspections included examination of the laminectomy site for evidence of infection and assessment of the hindlimbs for signs of autophagia or pressure.

Assessment of recovery from spinal cord contusion. Behavioral recovery was scored in an open field using the Basso, Beattie, and Bresnahan (BBB) scale (27), where a score of 0 registers complete paralysis and a score of 21 , complete mobility $(5,6,15,24)$. Blind scoring ensured that observers were not aware of the treatment received by individual rats. Approximately once a week, the locomotor activities of the trunk, tail, and hindlimbs were evaluated in an open field by placing each rat for 4 minutes in the center of a circular enclosure (90 cm diameter, $7 \mathrm{~cm}$ wall height) made of molded plastic with a smooth, nonslip floor. Before each evaluation, the rats were examined carefully for perineal infection, wounds in the hindlimbs, and tail and foot autophagia.

Retrograde labeling of rubrospinal neurons. Spinal contusion was preceded by immunization with spinal cord homogenate emulsified in CFA $(0.5 \mathrm{mg} / \mathrm{ml}$ bacteria $)$ or by injection with PBS in the same adjuvant. Three months later, two rats from each group were reanesthetized, and the dye rhodamine dextran amine (Fluoro-ruby; Molecular Probes, Eugene, Oregon, USA) was applied below the site of contusion at T12. After 5 days, 
the rats were again deeply anesthetized and their brains were excised, processed, and cryosectioned. Sections taken through the red nucleus were inspected and analyzed qualitatively and quantitatively by fluorescence and confocal microscopy.

Diffusion-anisotropy MRI. Diffusion anisotropy was measured in a DMX wide-bore spectrometer (Bruker, Karlsruhe, Germany), using a microscopy probe with a 5 -mm Helmholz coil and actively shielded magnetic field gradients. The observer was blinded to each rat's identity. Multislice echo imaging was performed with nine axial slices, with the central slice positioned at the center of the spinal injury. Images were obtained with a TE of 31 milliseconds, TR 2000 milliseconds, diffusion time 15 milliseconds, diffusion gradient duration 3 milliseconds, field of view $0.6 \mathrm{~cm}$, matrix size $128 \times 128$ pixels, slice thickness $0.5 \mathrm{~mm}$, and slice separation 1.18 $\mathrm{mm}$. Left to right images represent axial sections from head to foot. Four diffusion gradient values $(0,28,49$, and $71 \mathrm{~g} / \mathrm{cm}$ ) were applied along the read direction (transverse diffusion) or along the slice direction (longitudinal diffusion). Using an exponential fit for each pixel, we obtained a transverse and a longitudinal apparent diffusion coefficient (ADC) map, from which an anisotropy ratio matrix was derived. The accumulated anisotropy in each slice was integrated (28). For each rat, the lowest value of the slice anisotropy integral was defined as the lesion site.

\section{Results}

Spinal cord functional recovery after postinjury immunization with myelin basic protein. In light of our earlier finding that the therapeutic time window for ISCI is at least 1 week (15), and considering the rapid development of the $\mathrm{T}$ cell response after immunization, a $\mathrm{T}$ cell-based therapeutic active immunization could be considered. We first examined whether active immunization with MBP, immediately after contusion injury, can effectively replace the posttraumatic passive transfer of anti-MBP $\mathrm{T}$ cells. The initial choice of adjuvant was IFA, on the assumption that it would promote a cell-mediated immune response but would not cause $\operatorname{EAE}(29,30)$. Immunization with MBP emulsified in IFA, performed directly after severe ISCI, resulted in better recovery than that seen in control rats similarly injected with PBS in IFA. However, this postinjury immunization was not as effective as the immunization protocols described previously (preinjury active immunization with MBP in IFA [ref. 15] or postinjury passive transfer of $\mathrm{T}$ cells directed to MBP [Figure 1]). We reasoned that the difference was due to the choice of the adjuvant (IFA). This adjuvant induced a relatively delayed onset of the response to MBP, which, in turn, did not fit within the therapeutic time window for neuroprotection after spinal cord contusion injury (15). We performed a set of vaccinations in spinally contused rats to determine whether the use of MBP emulsified in CFA would reduce the time for evoking a $\mathrm{T}$ cell response relative to MBP emulsified in IFA. The findings confirmed that
CFA induces an earlier and stronger $T$ cell response than that induced by IFA. Therefore, in all subsequent immunization protocols, we used antigens emulsified in CFA. Active immunization with spinal cord homogenate emulsified in CFA. To determine whether the effectiveness of active immunization could be increased by a change of the antigen, we examined the effect of immunization with spinal cord homogenate, which contains a spectrum of myelin proteins, rather than with MBP only. Before examining a postinjury vaccination, we examined the efficacy of preinjury immunization. Seven days before spinal cord contusion, female Lewis rats $(n=7)$ were immunized with spinal cord homogenate emulsified in CFA (containing $0.5 \mathrm{mg} / \mathrm{ml}$ bacteria). A control group of female Lewis rats $(n=7)$ was injected with PBS emulsified in the same adjuvant. Three uninjured female rats that were immunized according to the same protocol showed no detectable symptoms of EAE. In a separate set of experiments, female rats $(n=6$ for each group) were immunized, 7 days before spinal cord contusion, with spinal cord homogenate emulsified in CFA containing $5 \mathrm{mg} / \mathrm{ml}$ bacteria. Because of slight variations in the results among contused control rats,

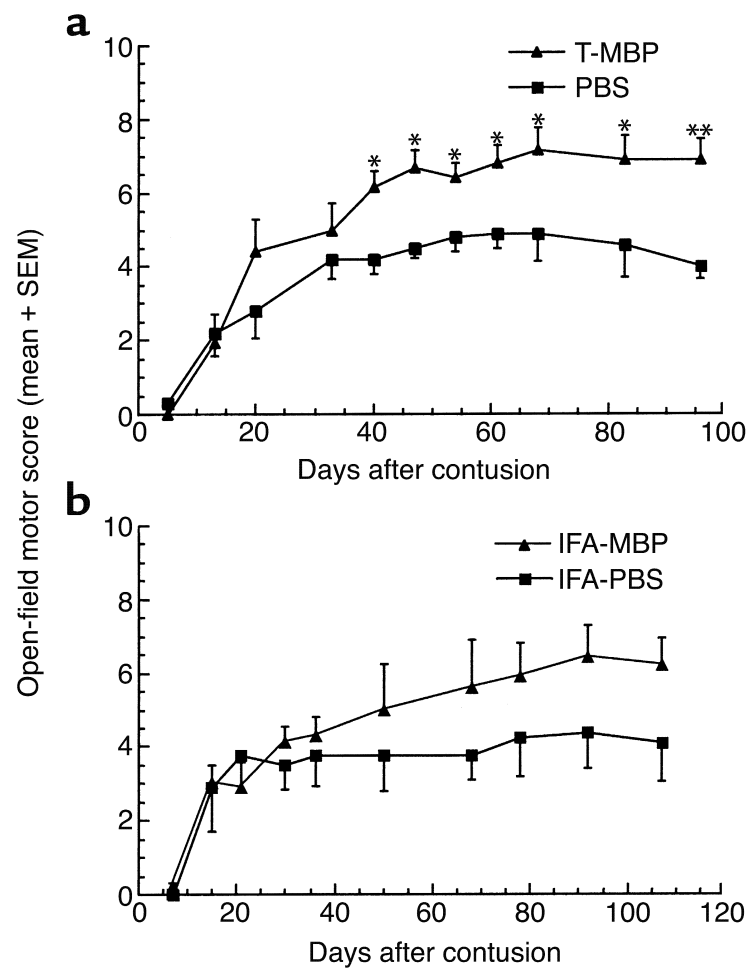

Figure 1

Posttraumatic passive and active immunization with MBP after spinal cord contusion. Passive transfer (a) of MBP-reactive T cells (T-MBP), immediately after spinal cord contusion, conferred significant neuroprotection in female Lewis rats $(n=6)$, whereas active immunization (b) with $\operatorname{MBP}(100 \mu \mathrm{g} / \mathrm{rat})$ emulsified in IFA, immediately after the contusion, had a smaller effect that was not statistically significant $(n=5)$. This result suggests that the autoimmune response evoked by posttraumatic vaccination with IFA is not sufficient (in its timing or extent) to significantly affect functional recovery after $\mathrm{ISCl}\left({ }^{*} P<0.05\right.$, ${ }^{*} P<0.01$, two-tailed Student's $t$ test). 


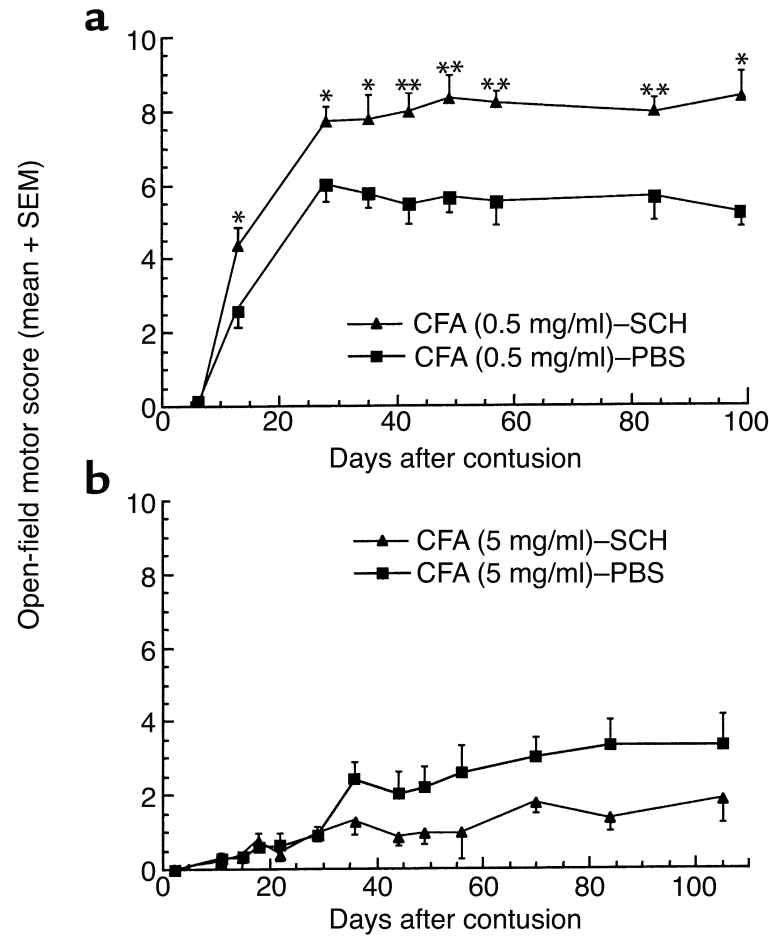

Figure 2

The type of adjuvant can affect the outcome of ISCI. (a) Seven female Lewis rats, immunized ( 7 days before contusion) with spinal cord homogenate $(\mathrm{SCH})$ emulsified in CFA containing $0.5 \mathrm{mg} / \mathrm{ml} \mathrm{M}$. butyricum, recovered significantly better $(P<0.05$, two-way ANOVA with replications; ${ }^{*} P<0.05,{ }^{*} P<0.01$, two-tailed Student's $t$ test) than did seven control littermates injected with PBS in the same adjuvant. (b) The same immunization protocol, when applied with a stronger adjuvant, has the opposite effect. Rats immunized with spinal cord homogenate in CFA containing $5 \mathrm{mg} / \mathrm{ml} \mathrm{M}$. tuberculosis developed severe EAE symptoms and showed significantly worse recovery than did six control littermates injected with PBS in the same adjuvant $(P<0.05$, two-way ANOVA with replications).

a control group of rats injected with an emulsion of PBS and the relevant adjuvant or of untreated contused rats was included in each experiment shown below.

Immunization of female Lewis rats with spinal cord homogenate emulsified in the adjuvant with the lower bacterial content $(0.5 \mathrm{mg} / \mathrm{ml})$ resulted in significantly better recovery from the ISCI than that obtained in PBStreated controls (Figure 2a): a maximal score of $8.2 \pm 0.2$ (mean $\pm \mathrm{SE}$ ) on the BBB locomotor rating scale compared with $5.5 \pm 0.2$ (two-way ANOVA with replications; $P<0.05)$. A BBB score of 8.2 indicates extensive movement of all three hindlimb joints and plantar placement of the paw (three animals showed occasional weight support and plantar steps), whereas a score of 5.5 indicates slight movement of two hindlimb joints and extensive movement of the third, without plantar placement of the paw or swiping and without weight support. After immunization with spinal cord homogenate emulsified in the more potent adjuvant (containing $5 \mathrm{mg} / \mathrm{ml} \mathrm{bac-}$ teria; Figure $2 \mathrm{~b}$ ), spinally contused rats $(n=6)$ showed no recovery relative to controls, and three noninjured lit- termates showed extremely severe symptoms of EAE. It should be noted that the control groups immunized with PBS in the two different adjuvants showed differences that might reflect a general effect of the immune response to trauma, possibly with some boosting by the bacteria. The observed loss of the beneficial effect, together with signs of severe encephalitogenicity when the bacterial dosage was high, raises questions about the connection between beneficial autoimmunity and development of autoimmune disease. It is possible that both protective and pathogenic autoimmunity depend on the presence of specific autoreactive $T$ cells and regulatory $T$ cells (22), which act together in a beneficial or in a harmful way depending on their dosage and their proportions. Accordingly, the present results suggest that immunization with myelin-associated antigens should be rigorously controlled in order to avoid pathogenicity and to promote neuroprotection.

Morphological evidence for spinal cord protection after active immunization. The behavioral results described above were correlated with results obtained by retrograde labeling of rubrospinal neurons in the red nucleus of the brain, after administration of the neurotracer dye Fluoro-ruby below the site of spinal cord contusion. Sections from red nuclei of rats that were immunized with spinal cord homogenate in CFA $(0.5 \mathrm{mg} / \mathrm{ml})$ or injected with PBS in the same adjuvant are shown in Figure 3. As previously reported (15), the number of stained rubrospinal neurons correlated well with the behavioral outcome as measured by the BBB score.

In the diffusion-anisotropy MRI analysis, axial anisotropy maps calculated from the diffusion weighted images taken from the spinal cords of rats immunized with spinal cord homogenate in CFA $(0.5 \mathrm{mg} / \mathrm{ml})$ showed areas of diffusion anisotropy along the entire length of the cord, and all cords manifested a continuous longitudinal structure (Figure 4). In contrast, slices taken from the PBS-injected controls showed a loss of organized structure at the center of the lesion site, and the area of diffusion anisotropy in most of the analyzed slices was relatively small (Figure 4). The sum of anisotropy (SAI) representing the anisotropy value at the site of the injury (the slice with lowest SAI) was almost twice as high in rats immunized with spinal cord homogenate as in PBS-injected controls $(290$ compared with 167 arbitrary units). The behavioral outcome correlated well with the MRI results: the higher the behavioral score, the larger the area of diffusion anisotropy found at the site of the lesion (28).

Active immunization limits paralysis after spinal injury in EAE-resistant Sprague-Dawley rats. We recently showed that in SPD rats, known to be resistant to induction of EAE, spinal cord contusion evokes an endogenous beneficial immune response (18). Our studies using the rat model of optic nerve injury have shown that resistant rats recover better than do susceptible rats (17). Here we found that the same is true with respect to ISCI: EAE-resistant SPD rats show better spontaneous recovery from spinal cord contusion of identical severity and spine level than 

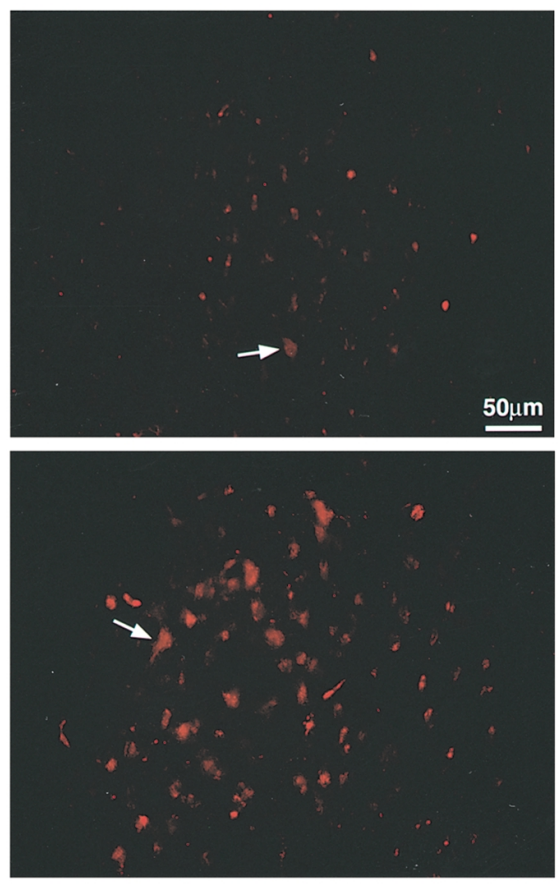

do susceptible Lewis rats (Figure 5). Accordingly, it was suggested that this endogenous protective immuneassociated response exists in EAE-resistant strains, but not in strains that are susceptible to EAE (17). It was therefore of interest to determine whether active immunization can further benefit the functional recovery in a strain that is spontaneously recovering better (i.e., EAEresistant), possibly by boosting the spontaneous protective response. This is an important issue, as the recovery by neuroprotection is inherently determined not only by the therapy, but also by the model and the amount of spared tissue that is vulnerable to secondary degeneration and amenable to neuroprotection. Twelve days before spinal cord contusion, five male SPD rats were immunized with spinal cord homogenate emulsified in CFA (containing $0.5 \mathrm{mg} / \mathrm{ml}$ bacteria) and five were injected with PBS in the same adjuvant (Figure 6). The

\section{Figure 3}

Retrograde labeling of cell bodies in the red nucleus. Spinal contusion was preceded 7 days earlier by immunization with spinal cord homogenate emulsified in CFA (containing $0.5 \mathrm{mg} / \mathrm{ml}$ bacteria) or by injection with PBS in the same adjuvant (Figure 2a). Three months later, two rats from each group were reanesthetized and the dye rhodamine dextran amine (Fluoro-ruby) was applied below the site of contusion. Five days later, the rats were sacrificed and their brains were excised, processed, and cryosectioned. Sections taken through the red nucleus were inspected and analyzed qualitatively and quantitatively by fluorescence and confocal microscopy. Significantly more labeled rubrospinal neurons were seen in slices from the immunized rats (bottom) (BBB score $=8$ ) than from the PBS-treated rats (top) $($ BBB score $=5.5)$.

immunized rats showed significantly better functional recovery than did the PBS-injected controls, starting from day 12 after contusion and at all indicated time points $(P<0.05$, two-way ANOVA with replications). These findings suggest that immunization with myelinassociated self-antigens can promote recovery after ISCI in both EAE-resistant and EAE-susceptible rats.

Our previous studies on injured rat spinal cord implied that immune neuroprotection by passive transfer of $\mathrm{T}$ cells directed to MBP can prevent complete paralysis after partial ISCI. David and colleagues suggested that vaccination with spinal cord homogenate emulsified in IFA before dorsal hemisection of the spinal cord could facilitate processes of axonal regeneration (31). It was consequently of interest to determine whether pre- or posttraumatic active immunization is effective in the event of complete transection. In this case, no axons maintain their integrity across the injury site, and thus any observed functional improvement below the primary site of transection would reflect regeneration rather than protection from the propagation of degeneration. Seven days before complete spinal cord transection, five female Lewis rats were immunized with spinal cord homogenate emulsified in IFA, and five were injected with PBS in IFA. Five more were immunized immediately after the complete transection. As in the case of passive immunization with

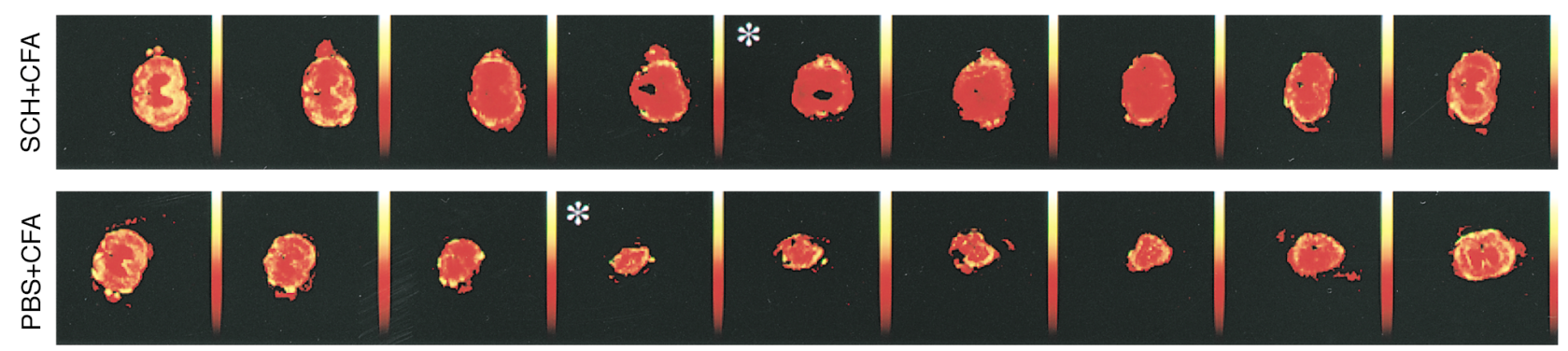

Figure 4

Maps showing diffusion anisotropy of the contused spinal cords. Rats were deeply anesthetized, and their excised spinal cords were immediately fixed and placed in 5-mm NMR tubes. Shown are representative maps of spinal cords of rats immunized with spinal cord homogenate and control rats, after contusion. Colors correspond to anisotropy ratios. The maps show the preservation of longitudinally ordered tissue at the lesion sites of the immunized rats. Note that the site of injury in the controls is much larger than in rats from the immunized group. The center of the injury site (asterisk) was determined by the slice with the lowest anisotropy value ( 290 arbitrary units in the immunized rat $[\mathrm{BBB}=8.5]$ and 167 units in the control rats $[\mathrm{BBB}=6])$. 


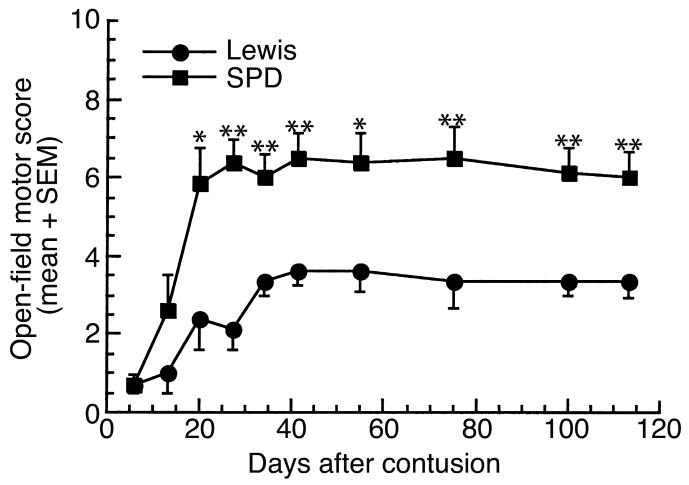

Figure 5

EAE-resistant rats display a better functional recovery than rats from an EAE-susceptible strain. Age- and weight-matched SPD and Lewis male rats $(n=5)$ suffered spinal cord contusion at level T9. Functional recovery was blindly scored using the BBB locomotor rating scale. SPD rats showed significantly better hindlimb locomotion starting from day 20 and at all time points examined thereafter ( ${ }^{*} P<0.05$; ${ }^{*} P<0.01$; two-tailed Student's $t$ test). This result supports our previous findings (see the text) suggesting that animals that are resistant to pathogenic autoimmunity are capable of mounting a spontaneous protective autoimmune response after CNS injury.

MBP-reactive T cells (15), none of these rats showed any significant locomotor function when examined weekly, up to 4 months after transection (data not shown).

Postinjury immunization with altered peptide ligands: neuroprotection with reduced risk of disease. Having observed that complete paralysis after ISCI can be prevented by active postinjury immunization with myelin-associated antigens, we proceeded to search for a safe (i.e., nonpathogenic) peptide for purposes of immunization. One way to develop an immunization protocol that will benefit the injured spinal cord and be safe in both susceptible and resistant strains might be to use an encephalitogenic attenuated peptide (e.g., an APL). Examples of such peptides are MBP-derived encephalitogenic peptides modified at their $\mathrm{T}$ cell receptor-binding site, making them still recognizable to the T cells but no longer pathogenic $(32,33)$. We used the encephalitogenic MBP peptide (amino acids 87-99) in which residue 91 (lysine) was replaced with glycine (G91) or residue 96 (proline) was replaced with alanine (A96). These peptides, which are nonencephalitogenic, were shown to regulate the proliferative response and significantly modify the cytokine secretion profile of encephalitogenic MBP 87-99-reactive T cells (32). We found that immunization after the ISCI with either of these APL peptides emulsified in CFA $(0.5 \mathrm{mg} / \mathrm{ml}$ bacteria) led to an enhanced recovery of motor activity in both Lewis and SPD rats. After severe spinal cord contusion, five female Lewis rats were immunized intradermally at the base of their tails with the APL G91 $(100 \mu \mathrm{g} / \mathrm{rat})$ in CFA $(0.5 \mathrm{mg} / \mathrm{ml}$ bacteria), and five littermates (control) were injected with PBS in the same adjuvant (Figure 7). Significantly better locomotor function was observed in the G91-immunized rats than in the controls $(P<0.05$, two-way ANOVA with replications). Three uninjured littermates immunized with G91 in CFA showed no EAE symptoms. In a second set of experiments, male SPD rats ( $n=6$ in each group) were immunized, directly after spinal cord contusion, with A96 $(100 \mu \mathrm{g} / \mathrm{rat}$ or $500 \mu \mathrm{g} / \mathrm{rat})$ in CFA $(0.5 \mathrm{mg} / \mathrm{ml}$ bacteria). Rats immunized with $100 \mu \mathrm{g}$ A96 performed significantly better than did PBS-injected controls, starting from day 15 after contusion and at all time points thereafter $(P<0.05$, two-way ANOVA with replications; Figure 8a). Interestingly, however, rats immunized with $500 \mu \mathrm{g}$ A96, rather than $100 \mu \mathrm{g}$, performed significantly worse than did PBS-injected controls $(P<0.05$, two-way ANOVA with replications; Figure 8b). This finding might provide a possible explanation for the exacerbation observed in patients with multiple sclerosis treated with extremely high doses of APLs (50 mg/week) (34).

\section{Discussion}

This study presents a novel therapeutic posttraumatic vaccination protocol for CNS injuries. Furthermore, the use of an altered encephalitogenic peptide in treating ISCI provides a way whereby a full benefit can be obtained without the risk of developing autoimmune disease, even in individuals that are susceptible to development of autoimmune diseases (e.g., multiple sclerosis). This strategy can therefore be used in translating this treatment into a clinically applicable therapy. The benefit of immunization is manifested by a significant improvement in the recovery of motor performance and can be achieved in rat strains that are prone to autoimmune disease development, as well as in those that are not. The observed differences in the rate of spontaneous recovery from ISCI between the strains appear to be attributable to the differences in their ability to sustain a $\mathrm{T}$ cell-dependent beneficial immune response (17).

The initial purpose of this work was to determine whether posttraumatic immunization can be as effective as passive transfer of $\mathrm{T}$ cells in promoting recovery

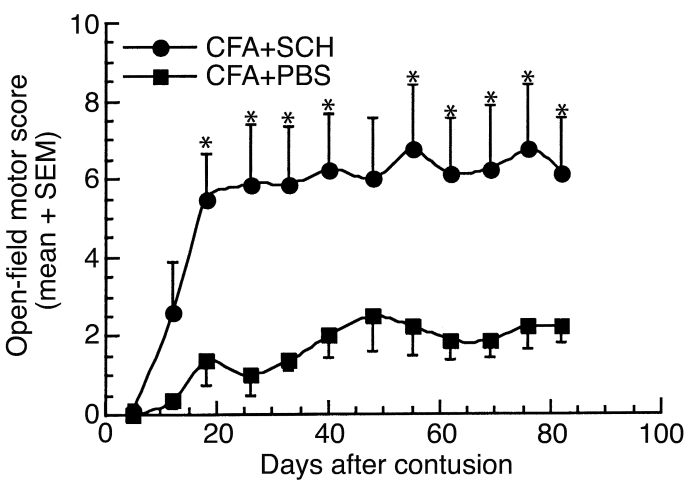

Figure 6

Functional outcome of spinal cord contusion in an EAE-resistant strain can be improved by active immunization. Five male SPD rats were immunized with spinal cord homogenate emulsified in CFA (containing $0.5 \mathrm{mg} / \mathrm{ml}$ bacteria), and five were injected with PBS in the same adjuvant. Twelve days later, the rats were subjected to spinal cord contusion and their locomotor behavior in an open field was scored at the indicated times. Significantly better recovery was observed in the immunized rats than in the PBS-treated controls $(P<0.05$, two-way ANOVA with replications; ${ }^{*} P<0.05$, two-tailed Student's $t$ test $)$. 


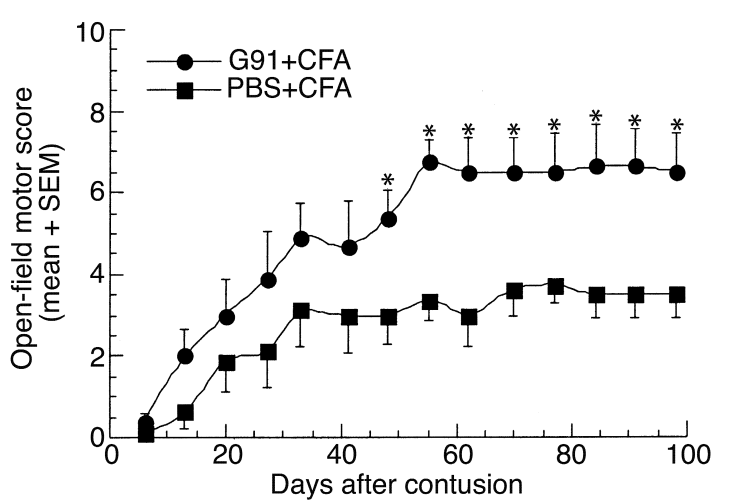

Figure 7

Immunization with a "safe," nonencephalitogenic, modified MBP peptide can promote recovery from spinal cord contusion. Five female Lewis rats were immunized, immediately after spinal cord contusion, with G91 peptide $(100 \mu \mathrm{g} / \mathrm{rat})$ emulsified in CFA (containing 0.5 $\mathrm{mg} / \mathrm{ml}$ bacteria). Five female littermates were subjected to spinal cord contusion and immediately injected with PBS in the same adjuvant. Significantly better functional recovery was observed in the immunized rats than in the PBS-treated controls $(P<0.05$, two-way ANOVA with replications; ${ }^{*} P<0.05$, two-tailed Student's $t$ test). Mean maximal locomotor score was $6.75 \pm 0.56$ in the G91-vaccinated rats $(n=5)$, $3.75 \pm 0.46$ in the PBS-CFA-injected rats $(n=5)$, and $1.5 \pm 0.8$ in the PBS-injected rats ( $n=6$, data not shown in the figure).

after ISCI. We then strove to find a way to avoid the risk of autoimmune disease while maintaining the full benefit of the posttraumatic immunization. The effects of different immunization protocols were examined by injecting rats with MBP or spinal cord homogenate emulsified in IFA or in CFA containing different amounts of bacteria. Finding that the concentration of the adjuvant can affect the functional outcome of the injury suggests a delicate balance between beneficial and pathogenic autoimmunity (both physiologically and after therapeutic intervention), which is influenced not only by the antigen, but also by the adjuvant. Moreover, a potent adjuvant by itself may affect the outcome by boosting the endogenous response, with opposing effects in genetically different strains. An adjuvant with a relatively large bacterial component might produce a response that would mask, compete with, or augment the response against the coinjected myelin antigen. This response might also affect the endogenous physiological neuroprotective response. Indeed, our earlier studies using the optic nerve model have implied the possibility that an adjuvant containing high bacterial concentration may boost the endogenous protective response in resistant strains and worsen the endogenous outcome in susceptible strains (17). Recently, we attempted to find a correlation between protective autoimmunity and extent of inflammation observed in and around the lesion site, using immunocytochemical staining procedures. Interestingly, the presence of macrophages and $\mathrm{T}$ cells did not necessarily provide a resolution with respect to the positive or negative contribution of these cells to the overall outcome of the insult. The phenotype of the cells and their antigenic specificity (related to $\mathrm{T}$ cells) are currently under investigation.

We have previously shown, using models of ISCI or optic nerve injury, that the timing, type, and strength of the autoimmune response are crucial factors in determining the outcome of treatment. For example, immunization with the encephalitogenic peptide PLP139-151 in a mouse model of optic nerve crush was found beneficial only when the EAE symptoms it induced were mild (21). Likewise, using a protocol that resulted in very severe symptoms of EAE had a highly unfavorable outcome in injured rats (present work; E. Hauben et al., unpublished observations). Immunization with a very high dosage of antigen might also be ineffective or destructive because the resulting anergy might eliminate the response against the antigen and perhaps also suppress the physiological neuroprotection. These observations are in line with our current suggestion that

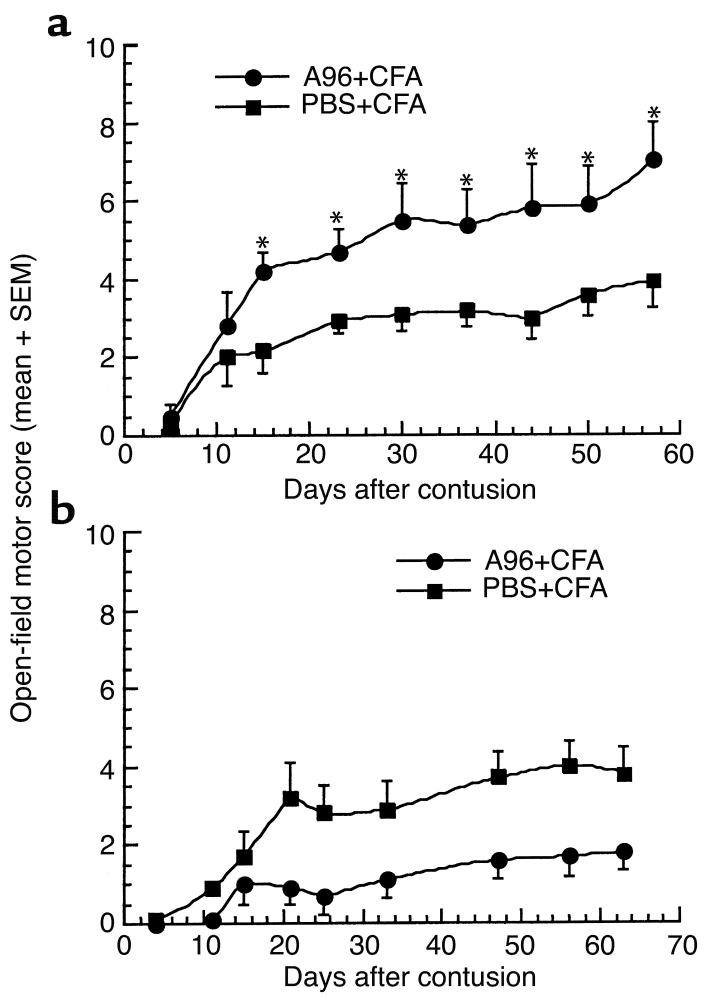

Figure 8

Immunization after ISCI with a modified MBP peptide promotes functional recovery in EAE-resistant SPD rats. (a) Six SPD male rats were immunized, immediately after spinal cord contusion, with A96 peptide $(100 \mu \mathrm{g} / \mathrm{rat})$ in CFA (containing $0.5 \mathrm{mg} / \mathrm{ml}$ bacteria), and six littermates were injected with PBS in the same adjuvant. Rats immunized with A96 recovered significantly better than PBS-injected controls $\left(P<0.05\right.$, two-way ANOVA with replications; ${ }^{*} P<0.05$, two-tailed Student's $t$ test). Mean maximal locomotor score was $7 \pm$ 0.96 in the A96-vaccinated rats $(n=5), 3.9 \pm 0.45$ in the PBSCFA-injected rats $(n=5)$, and $3.0 \pm 0.8$ in the PBS-injected rats ( $n=6$, data not shown in the figure). (b) Immunization of SPD male rats $(n=5)$, immediately after spinal cord contusion, with A96 peptide $(500 \mu \mathrm{g} / \mathrm{rat})$ in CFA (containing $0.5 \mathrm{mg} / \mathrm{ml}$ bacteria) had a significant negative effect on functional recovery $(P<0.05$, two-way ANOVA with replications). 
protective autoimmunity depends on the availability of autoimmune $\mathrm{T}$ cells and regulatory $\mathrm{T}$ cells, the nature of which are currently being investigated $(18,22,23)$. These observations should lead to a resolution of the long debate as to whether inflammation is bad and should be avoided or good and should be augmented; the answer being that it should be modulated and optimized in time and amount to yield the optimal benefit. Studies in our laboratory have shown that CNS injury evokes a physiological $\mathrm{T}$ cell-mediated immune response that can improve the spontaneous outcome of a second injury to another CNS site and can be transferred from spinally injured rats via ex vivo MBP-activated splenocytes to newly injured rats with resulting improvement in their functional outcome (18). In a more recent study, it was demonstrated that the survival rate of retinal ganglion cells after crush injury to the optic nerve or glutamate insult to the retinal ganglion cells is significantly higher in rat and mouse strains that are resistant to EAE than in susceptible strains $(17,35)$. This difference could not be detected in adult rats that were thymectomized soon after birth and therefore lacked immune responses mediated by $T$ cells (17). Taken together, these findings suggest that in resistant strains, the physiological $\mathrm{T}$ cell-dependent immune response, which spontaneously occurs after CNS trauma, significantly improves recovery. However, in susceptible strains, there is either no such naturally occurring response, or the response occurs at a nonoptimal time, and the outcome of CNS injury is therefore relatively poor. Our present findings show that active immunization, even in EAE-resistant SPD rats, yielded significant boosting of the neuroprotective effect. It appears that EAE-susceptible strains lack the control mechanism that promotes the physiological beneficial autoimmune response, but that this response can, nevertheless, be induced by immunization, depending on the choice of antigen and adjuvant.

In searching among the CNS injury-associated proteins for a "safe" (nonpathogenic) antigen for therapeutic immunization, we examined the posttraumatic effect of a peptide, which, although originally encephalitogenic, was modified by the replacement of a single amino acid in its $\mathrm{T}$ cell receptor-binding site (a manipulation that ameliorated the pathogenic effect). The mechanism whereby APLs diminish encephalitogenicity is still not fully understood. It was proposed that these peptides deliver a dominant negative signal to a subpopulation of T cells (36). Other studies have proposed that the APLs change the polarity of the autoreactive $T$ cells from Th1 to Th2 $(32,37)$ or alter cytokine production (38). The general notion is that APLs provide a partial $\mathrm{T}$ cell signaling (39). Immunization with APLs in our experimental paradigm may be viewed as analogous to vaccination against invading microorganisms using an inoculum consisting of their nonvirulent forms. It is possible that vaccination with APLs induces the production of antigen-specific regulatory $\mathrm{T}$ cells without inducing proliferation of encephalitogenic T cells (40).
We found that a single vaccination with APLs emulsified in CFA, given immediately after spinal cord contusion, triggers boosting of the endogenous neuroprotective effect in resistant strains and induction of this effect in susceptible strains. Given that the use of APLs in CFA is not accompanied by the risk of autoimmune disease, even in susceptible strains, it is possible to use this approach in searching for a therapeutic vaccination for ISCI. Recent studies have demonstrated that repeated use of such peptides in high doses may not be safe in some patients with severe multiple sclerosis (34). However, when patients with multiple sclerosis were given a single dose, no adverse effects were reported. In fact, administration of lower doses of this altered peptide reduces the extent of inflammation on MRI scans, apparently without exacerbating the disease (41).

Further studies are needed to identify or develop a safe peptide that can be used by all individuals, whether or not they are susceptible to autoimmune disease development. The synthetic oligopeptide Copolymer-1 (Cop-1) has already been found by our group to yield promising results in rats with optic nerve injury (35, 42). This copolymer, which is known to be nonencephalitogenic, has been approved by the FDA as an immunosuppressive drug for the treatment of multiple sclerosis $(42,43)$. The observed benefit in using the altered peptides and Cop-1 may result from boosting of the regulatory arm of the protective autoimmune network (22), or partial activation of the autoimmune $\mathrm{T}$ cells (39), both of which are apparently essential for the protective autoimmunity (22). This issue is under investigation in our laboratory.

In the past, posttraumatic inflammation was viewed as destructive, and therapy based on the use of anti-inflammatory drugs was therefore generally regarded as beneficial. Our studies, which suggest that immune cells are part of the body's own repair mechanism even in cases of CNS trauma, argue against immunosuppression and encourage immunomodulation aimed at increasing the beneficial effect of the immune system while reducing undesirable side effects. Recently we demonstrated that the use of anti-inflammatory drugs in combination with the present strategy of active vaccination abolished the benefit of the vaccination under conditions where the anti-inflammatory drugs by themselves had no effect (E. Hauben et al., unpublished results).

The results of this study show that in rats with severe spinal contusion, complete paralysis can be prevented and recovery thus promoted by posttraumatic immunization with synthetic peptides. They also show that both susceptible and resistant strains can benefit from postinjury immunization, and that the beneficial effect on neuroprotection is influenced by the choice of both the peptide and the adjuvant.

\section{Acknowledgments}

We thank S. Smith for editing of the manuscript, and A. Abramov for animal maintenance. M. Schwartz holds the Maurice and Ilse Katz Professorial Chair in 
Neuroimmunology. The work was supported by Proneuron Ltd. and in part by grants from The Glaucoma Research Foundation and Alan Brown Foundation for Spinal Cord Injury awarded to M. Schwartz.

1. Bazan, N.G., Rodriguez de Turco, E.B., and Allan, G. 1995. Mediators of injury in neurotrauma: intracellular signal transduction and gene expression. J. Neurotrauma. 12:791-814.

2. Faden, A.I. 1993. Experimental neurobiology of central nervous system trauma. Crit. Rev. Neurobiol. 7:175-186.

3. Liu, D., Yang, R., Yan, X., and McAdoo, D.J. 1994. Hydroxyl radicals generated in vivo kill neurons in the rat spinal cord: electrophysiological, histological, and neurochemical results. J. Neurochem. 62:37-44.

4. Moalem, G., et al. 1999. Autoimmune T cells protect neurons from secondary degeneration after central nervous system axotomy. Nat. Med. 5:49-55.

5. Hauben, E., et al. 2000. Autoimmune T cells as potential neuroprotective therapy for spinal cord injury. Lancet. 355:286-287.

6. Basso, D.M., Beattie, M.S., and Bresnahan, J.C. 1996. Graded histologi$\mathrm{cal}$ and locomotor outcomes after spinal cord contusion using the NYU weight-drop device versus transection. Exp. Neurol. 139:244-256.

7. Bavetta, S., Hamlyn, P.J., Burnstock, G., Lieberman, A.R., and Anderson, P.N. 1999. The effects of FK506 on dorsal column axons following spinal cord injury in adult rats: neuroprotection and local regeneration. Exp. Neurol. 158:382-393.

8. Beattie, M.S., et al. 1997. Endogenous repair after spinal cord contusion injuries in the rat. Exp. Neurol. 148:453-463.

9. Behrmann, D.L., Bresnahan, J.C., and Beattie, M.S. 1994. Modeling of acute spinal cord injury in the rat: neuroprotection and enhanced recovery with methylprednisolone, U-74006F and YM-14673. Exp. Neurol. 126:61-75.

10. Bethea, J.R., et al. 1999. Systemically administered interleukin-10 reduces tumor necrosis factor-alpha production and significantly improves functional recovery following traumatic spinal cord injury in rats. J. Neurotrauma. 16:851-863.

11. Brewer, K.L., Bethea, J.R., and Yezierski, R.P. 1999. Neuroprotective effects of interleukin-10 following excitotoxic spinal cord injury. Exp. Neurol. 159:484-493.

12. Constantini, S., and Young, W. 1994. The effects of methylprednisolone and the ganglioside GM1 on acute spinal cord injury in rats. J. Neurosurg. 80:97-111.

13. Crowe, M.J., Bresnahan, J.C., Shuman, S.L., Masters, J.N., and Beattie, M.S. 1997. Apoptosis and delayed degeneration after spinal cord injury in rats and monkeys [erratum 1997, 3:240]. Nat. Med. 3:73-76.

14. Yong, C., et al. 1998. Apoptosis in cellular compartments of rat spinal cord after severe contusion injury. J. Neurotrauma. 15:459-472.

15. Hauben, E., et al. 2000. Passive or active immunization with myelin basic protein promotes recovery from spinal cord contusion. J. Neurosci. 20:6421-6430.

16. Schwartz, M., Moalem, G., Leibowitz-Amit, R., and Cohen, I.R. 1999. Innate and adaptive immune responses can be beneficial for CNS repair. Trends Neurosci. 22:295-299.

17. Kipnis, J., et al. 2001. Neuronal survival after CNS insult is determined by a genetically encoded autoimmune response. J. Neurosci. 21:4564-4571.

18. Yoles, E., et al. 2001. Protective autoimmunity is a physiological response to CNS trauma. J. Neurosci. 21:3740-3748.

19. Cohen, I.R., and Schwartz, M. 1999. Autoimmune maintenance and neuroprotection of the central nervous system. J. Neuroimmunol. 100:111-114.

20. Schwartz, M., and Cohen, I.R. 2000. Autoimmunity can benefit selfmaintenance. Immunol. Today. 21:265-268.

21. Fisher, J., et al. 2001. Vaccination for neuroprotection in the mouse optic nerve: implications for optic neuropathies. J. Neurosci. 21:136-142.

22. Schwartz, M., and Kipnis, J. 2001. Protective autoimmunity: regulation and prospect for vaccination after brain and spinal cord injuries. Trends Mol. Med. 7:252-258.

23. Butovsky, O., Hauben, E., and Schwartz, M. 2001. Morphological aspects of spinal cord autoimmune neuroprotection-colocalization of T cells with B7-2 (CD86 and prevention of cyst formation. FASEB J. 15:1065-1067.

24. Young, W. 1996. Spinal cord regeneration. Science. 273:451.

25. Rapalino, O., et al. 1998. Implantation of stimulated homologous macrophages results in partial recovery of paraplegic rats. Nat. Med. 4:814-821.

26. Ben-Nun, A., and Cohen, I.R. 1982. Experimental autoimmune encephalomyelitis (EAE) mediated by $\mathrm{T}$ cell lines: process of selection of lines and characterization of the cells. J. Immunol. 129:303-308.

27. Basso, D.M., Beattie, M.S., and Bresnahan, J.C. 1995. A sensitive and reliable locomotor rating scale for open field testing in rats. J. Neurotrauma. 12:1-21.

28. Nevo, U., et al. 2001. Diffusion anisotropy MRI for quantitative assessment of recovery in injured rat spinal cord. Magn. Reson. Med. 45:1-9.

29. Killen, J.A., and Swanborg, R.H. 1982. Autoimmune effector cells. III. Role of adjuvant and accessory cells in the in vitro induction of autoimmune encephalomyelitis. J. Immunol. 129:759-763.

30. Namikawa, T., Richert, J.R., Driscoll, B.F., Kies, M.W., and Alvord, E.C., Jr. 1982. Transfer of allergic encephalomyelitis with spleen cells from donors sensitized with myelin basic protein in incomplete Freund's adjuvant. J. Immunol. 128:932-934.

31. Huang, D.W., McKerracher, L., Braun, P.E., and David, S. 1999. A therapeutic vaccine approach to stimulate axon regeneration in the adult mammalian spinal cord. Neuron. 24:639-647.

32. Gaur, A., et al. 1997. Amelioration of relapsing experimental autoimmune encephalomyelitis with altered myelin basic protein peptides involves different cellular mechanisms. J. Neuroimmunol. 74:149-158.

33. Vergelli, M., et al. 1996. Differential activation of human autoreactive T cell clones by altered peptide ligands derived from myelin basic protein peptide (87-99). Eur. J. Immunol. 26:2624-2634.

34. Bielekova, B., et al. 2000. Encephalitogenic potential of the myelin basic protein peptide (amino acids 83-99) in multiple sclerosis: results of a phase II clinical trial with an altered peptide ligand. Nat. Med. 6:1167-1175.

35. Schori, H., Yoles, E., and Schwartz, M. 2001. Autoimmunity counteracts the potential toxicity of physiological compounds in the central nervous system. J. Neuroimmunology. In press.

36. Smilek, D.E., et al. 1991. A single amino acid change in a myelin basic protein peptide confers the capacity to prevent rather than induce experimental autoimmune encephalomyelitis. Proc. Natl. Acad. Sci. USA. 88:9633-9637.

37. Nicholson, L.B., Greer, J.M., Sobel, R.A., Lees, M.B., and Kuchroo, V.K. 1995. An altered peptide ligand mediates immune deviation and prevents autoimmune encephalomyelitis. Immunity. 3:397-405.

38. Windhagen, A., et al. 1995. Modulation of cytokine patterns of human autoreactive $\mathrm{T}$ cell clones by a single amino acid substitution of their peptide ligand. Immunity. 2:373-380.

39. Sloan-Lancaster, J., and Allen, P.M. 1996. Altered peptide ligand-induced partial $\mathrm{T}$ cell activation: molecular mechanisms and role in $\mathrm{T}$ cell biology. Ann. Rev. Immunol. 14:1-27.

40. Nicholson, L.B., et al. 1998. Heteroclitic proliferative responses and changes in cytokine profile induced by altered peptides: implications for autoimmunity. Proc. Natl. Acad. Sci. USA. 95:264-269.

41. Kappos, L., et al. 2000. Induction of a non-encephalitogenic type $2 \mathrm{~T}$ helper-cell autoimmune response in multiple sclerosis after administration of an altered peptide ligand in a placebo-controlled, randomized phase II trial. Nat. Med. 6:1176-1182.

42. Kipnis, J., et al. 2000. T cell immunity to copolymer 1 confers neuroprotection on the damaged optic nerve: possible therapy for optic neuropathies. Proc. Natl. Acad. Sci. USA. 97:7446-7451.

43. Schori, H., et al. 2001. Vaccination for protection of retinal ganglion cells against death from glutamate cytotoxicity and ocular hypertension: implications for glaucoma. Proc. Natl. Acad. Sci. USA. 98:3398-3403. 